\title{
Dual-Band Magneto-Electric Dipole Antenna with High- Gain for Base-Station Applications
}

\author{
S. Khorasani ${ }^{1}$, J. Nourinia ${ }^{1}$, Ch. Ghobadi ${ }^{1}$, M. Shokri ${ }^{1}$, A. Hatamian ${ }^{1}$, and B. Virdee ${ }^{2}$ \\ ${ }^{1}$ Department of Electrical Engineering, Urmia University, Urmia, Iran \\ ${ }^{2}$ Center for Communications Technology, School of Computing \& Digital Media, London Metropolitan \\ University, London, United Kingdom \\ Corresponding author: majed.shokri@gmail.com
}

\begin{abstract}
:
The paper presents the results of a novel high-gain dual-band magneto-electric dipole (MED) antenna. The antenna comprises two pairs of horizontal metal plates of different heights that are excited by a $\Gamma$-shaped feedline structure. The antenna is entirely made of metal plates. Compared to traditional MED antennas the proposed design exhibits dual-band operation with higher radiation gain and whose frequency ratio can be modified by simply adjusting the heights of the two magneto-electric dipole segments. This feature is necessary for cellular base-station applications. The operation and characteristics of the antenna are validated by measurements. The measured results confirm the proposed antenna achieves fractional bandwidths of $13.31 \%$ (801.9916.2 MHz) and 19.76\% (1710.7-2085.7 MHz) for $\mathrm{S}_{11} \leq-10 \mathrm{~dB}$. It has stable unidirectional radiation patterns and optimum radiation gains of $9.2 \mathrm{dBi}$ and $7.8 \mathrm{dBi}$ at the first and second operating bands, respectively.
\end{abstract}

Key words: dual-band antenna, magneto-electric dipole, base station applications.

\section{Introduction:}

The fundamental MED antenna was first introduced in 2006 [1]. This type of antenna is based on the complementary concepts and composed of a shorted patch and an electric dipole. Due to excellent impedance matching and good radiation behavior, the MED antennas has recently attracted much interest [2-4]. In [2] a circularly polarized MED antenna is proposed that consists of two cross-placed magneto-electric dipoles and a feeding structure. The antenna has a fractional bandwidth of $85.7 \%$ ( 2 to $5 \mathrm{GHz}$ ), and a peak gain of approximately $8.3 \mathrm{dBi}$. Another design example of the MED antenna is investigated in [3] for cellular base stations. This antenna comprises a horizontal slotted electric dipole and a vertical magnetic dipole, which is shorted to the ground. This antenna has a fractional bandwidth of $63.3 \%(2.05$ to $3.95 \mathrm{GHz})$, and an average gain of $5 \mathrm{dBi}$. A cavity-backed MED antenna in [4] is employed in the implementation of a $4 \times 4$ antenna array. The antenna employs substrate integrated waveguide (SIW) aperture as a feed mechanism. This antenna is shown to achieve a fractional bandwidth of $19.1 \%$ (30.3 to $36.7 \mathrm{GHz})$ and a peak gain of about $20 \mathrm{dBi}$. The aforementioned antennas operate over a single frequency band. 
Next generation of wireless communication systems use software-defined radio architecture to enable base-stations to simultaneously support multiple radio standards. Consequently, there is a demand for antennas that are capable of dual-band and/or multiband operation. The use of dualband antennas decreases the number of antenna elements employed and reduces the cost and installation space for the base-station system. In the last few years, many studies have been carried out on the dual-band MED antennas [5-6]. In [5], a dual-layer structure is used to realize dualband MED antennas that have fractional bandwidths of $47.5 \%(1.70-2.76 \mathrm{GHz})$ and $30.2 \%(4.50-$ $6.10 \mathrm{GHz}$ ), and corresponding peak gains of $9.7 \mathrm{dBi}$ and $8.5 \mathrm{dBi}$, respectively. In [6], two Ushaped slots etched on the dipole results in dual-band characteristics covering $2.13-2.62 \mathrm{GHz}$ and 4.57-6.28 GHz with a corresponding peak gain of $4.7 \mathrm{dBi}$ and $3 \mathrm{dBi}$, respectively.

In this paper, we present a novel high-gain magneto-electric dipole antenna that exhibits dual-band characteristics and radiates energy unidirectionally in both bands. The gain performance in both bands is relatively high compared to other MED antennas. The proposed antenna is composed of two pairs of horizontal metal plates of different heights that are excited by a $\Gamma$-shaped metal strip. In fact, the upper metal plates determine the frequency of the first band, and the lower metal plate the second frequency band. The proposed antenna is a simple structure that can be easily designed and fabricated.

\section{Single-Band MED Antenna Design}

Initially a single-band MED antenna was designed, which is based on the traditional MED antenna structure. The geometry of the single-band MED antenna is shown in Fig.1. It consists of two horizontal metal plates that are placed above the metallic ground-plane at a height of $\sim \lambda / 4$, where $\lambda$ is the wavelength in the free-space at the center of the operating frequency band. The plates are connected to the two vertical short-circuited patched. Horizontal plates act like an electric dipole and the vertical plates as a magnetic dipole. The length of the electric and magnetic dipole is designed close to a quarter-wavelength, corresponding to the desired resonant frequency. A ' $\Gamma$-shaped' feed strip is used to excite the electric and magnetic dipoles. The feed strip is connected to the inner pin of the SMA connector that is located under the metallic ground-plane. The equivalent circuit of the single-band MED antenna is shown in Fig.1(d). The feedline is represented by inductor $L_{f}$ and coupling capacitance between feedline and shorted vertical patches by $C_{f}$. The electric dipole consists of resistor $R_{d}$ is in series with an inductor $L_{d}$ and a capacitor $C_{d}$, whereas a resistor $R_{m}$ is in parallel with an inductor $L_{m}$ and a capacitor $C_{m}$ represents the magnetic dipole.

A parametric analysis was conducted to understand of how the $\Gamma$-shaped feed strip affected the $\mathrm{S}_{11}$ performance of the single-band MED antenna. The results of this analysis in Fig.2 shows the frequency of the peak dip in $\mathrm{S}_{11}$ decreases from $1950 \mathrm{MHz}$ to $1805 \mathrm{MHz}$ with increase in $L_{1}$ length from $24.5 \mathrm{~mm}$ to $32.5 \mathrm{~mm}$; however, there is negligible change in the impedance bandwidth. Simulated reflection-coefficient $\left(\mathrm{S}_{11}\right)$ and peak gain of this antenna for $L_{1}=27.5 \mathrm{~mm}$ are shown in Fig.3. The single-band MED antenna has a fractional bandwidth of $18.73 \%$ (1708.5 to 2061.6 $\mathrm{MHz}$ ) for $\mathrm{S}_{11} \leq-10 \mathrm{~dB}$, and average gain of approximately $8 \mathrm{dBi}$. 


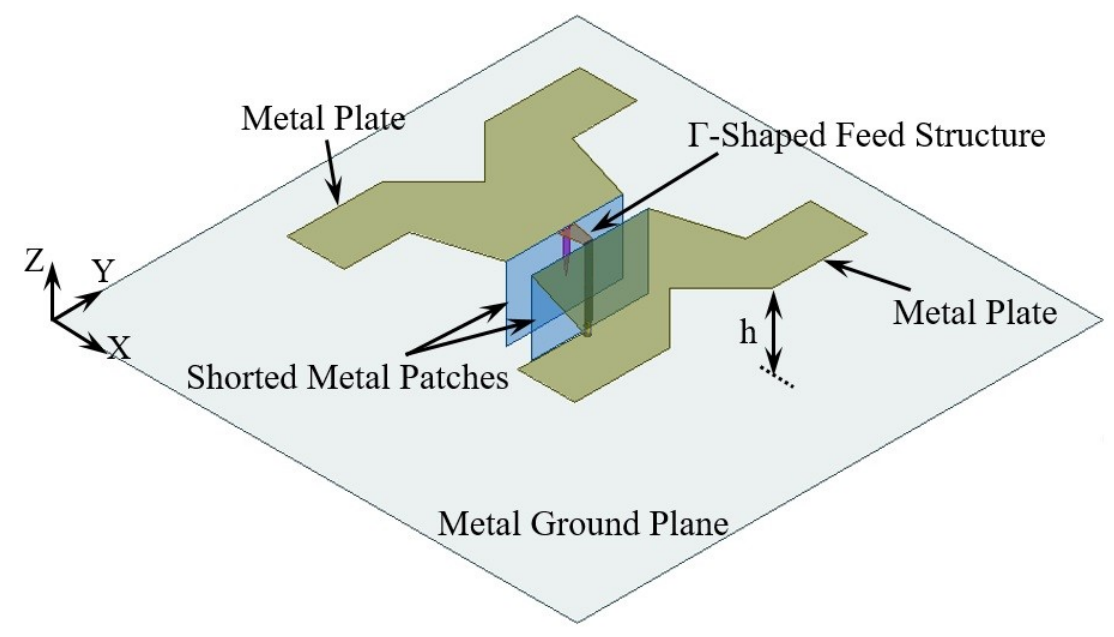

(a)

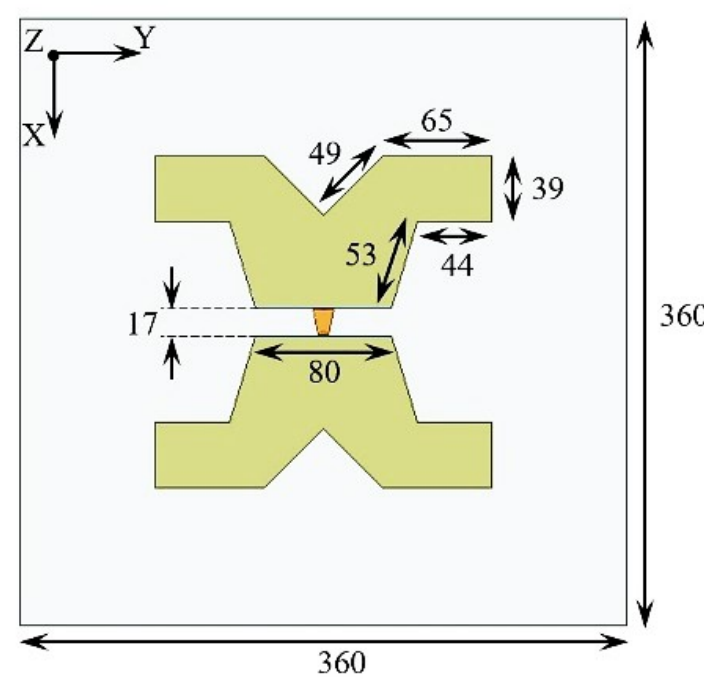

(b)

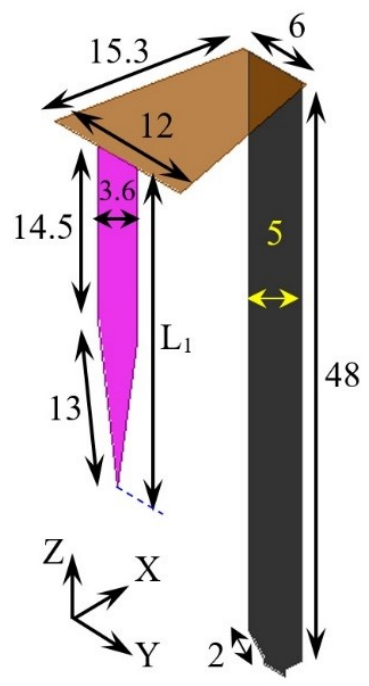

(c)

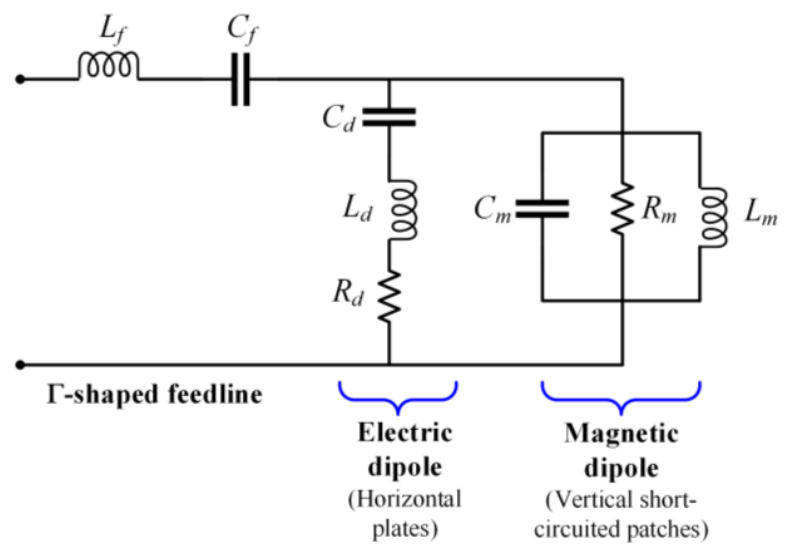

(d) 
Fig. 1 Structure of the single-band MED antenna and $\Gamma$-shaped feed structure with the antenna results. a) Perspective view, $(h=50 \mathrm{~mm})$, b) Top view, c) $\Gamma$-shaped feed structure, $\left(L_{l}=27.5 \mathrm{~mm}\right), \&$ d) Equivalent circuit of the singleband MED antenna. (units: $\mathrm{mm}$ )

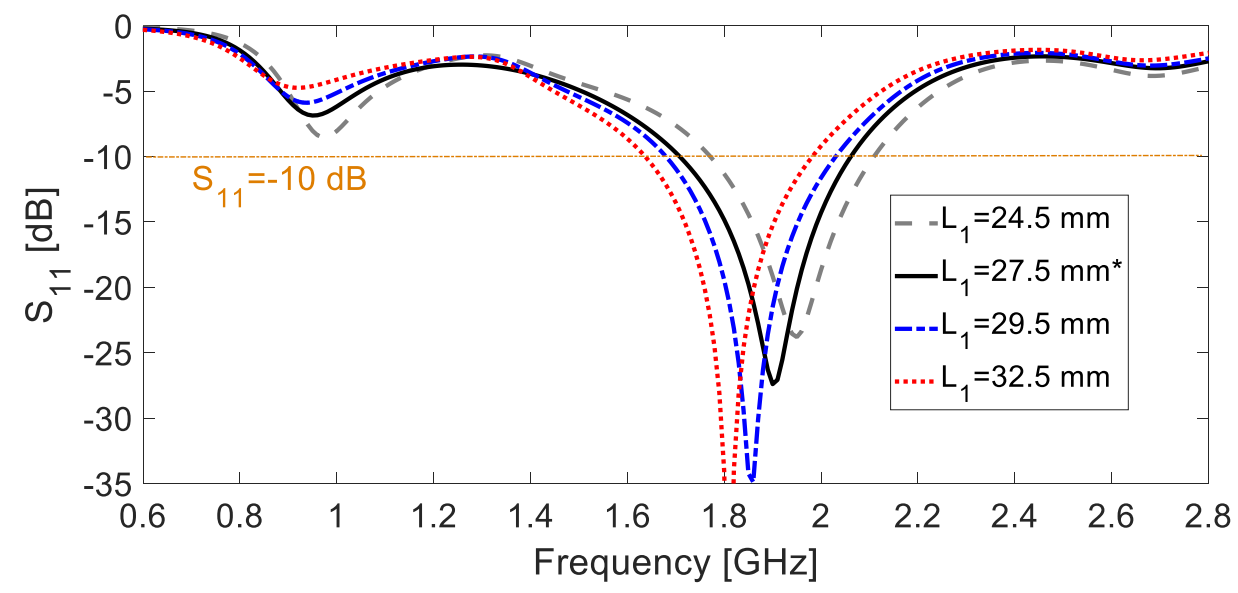

Fig. 2 Simulated reflection-coefficient $\left(\mathrm{S}_{11}\right)$ response of the single-band MED antenna as a function of $\Gamma$-shaped feed strip length $L_{l}$.

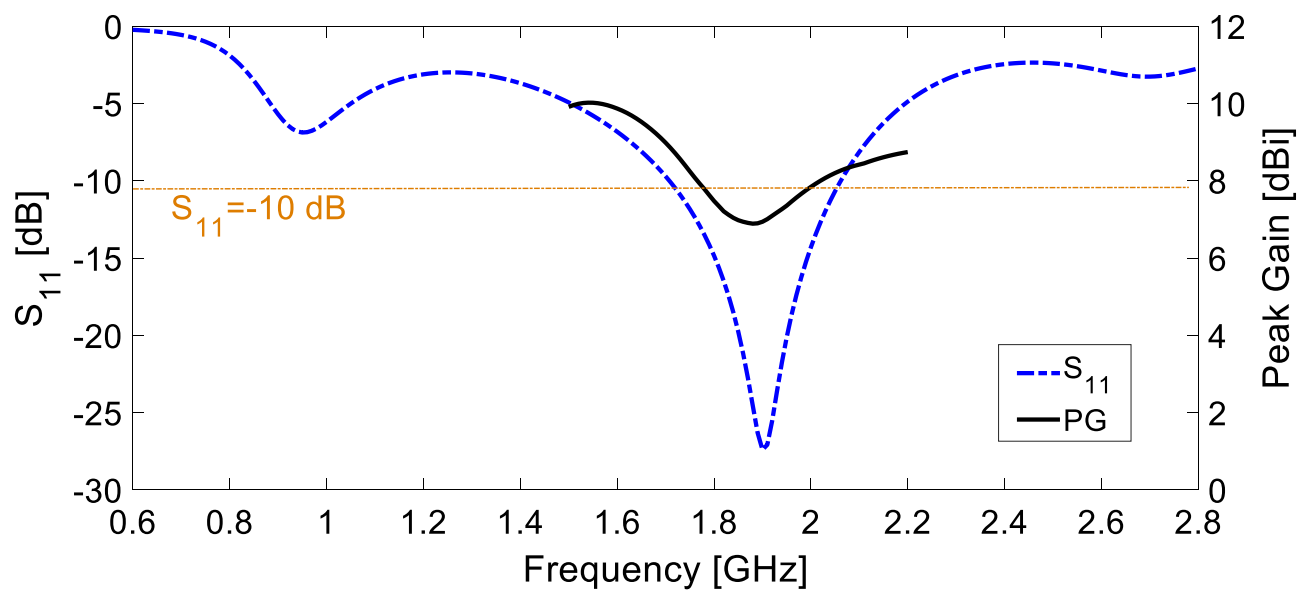

Fig. 3 Simulated reflection-coefficient $\left(\mathrm{S}_{11}\right)$ and peak gain response of the single-band MED antenna for $L_{1}=27.5$ $\mathrm{mm}$.

\section{Proposed Dual-Band MED Antenna Design}

The structure of the proposed dual-band MED antenna is shown in Fig.4. Compared to the singleband antenna structure in Fig.1 each of the horizontal metal plates is divided into two equal segments which are of different heights. Consequently, there are two pairs of horizontal metal plates. To generate two distinct frequency bands, one pair of the metal plates is located at the height of $h_{1}$, and the other one positioned at the height of $h_{2}$. The frequency ratio between the two bands is determined by the gap between the segment heights. The equivalent circuit of the dualband MED antenna is shown in Fig.4(c). The gap between the pair of horizontal plates for the 
desired operating band in this case is $25 \mathrm{~mm}$. A single band antenna can be easily realized by making $h_{1}=h_{2}$.

The current distribution over the antenna at its upper and lower operating bands in Fig.5 shows the current to be concentrated predominately at the $\Gamma$-shaped feed structure, which consists of three segments. This is not surprising as it is connected to the SMA excitation point. The coupled current over the vertical shorted patch is more intensely distributed than at the horizontal plates.

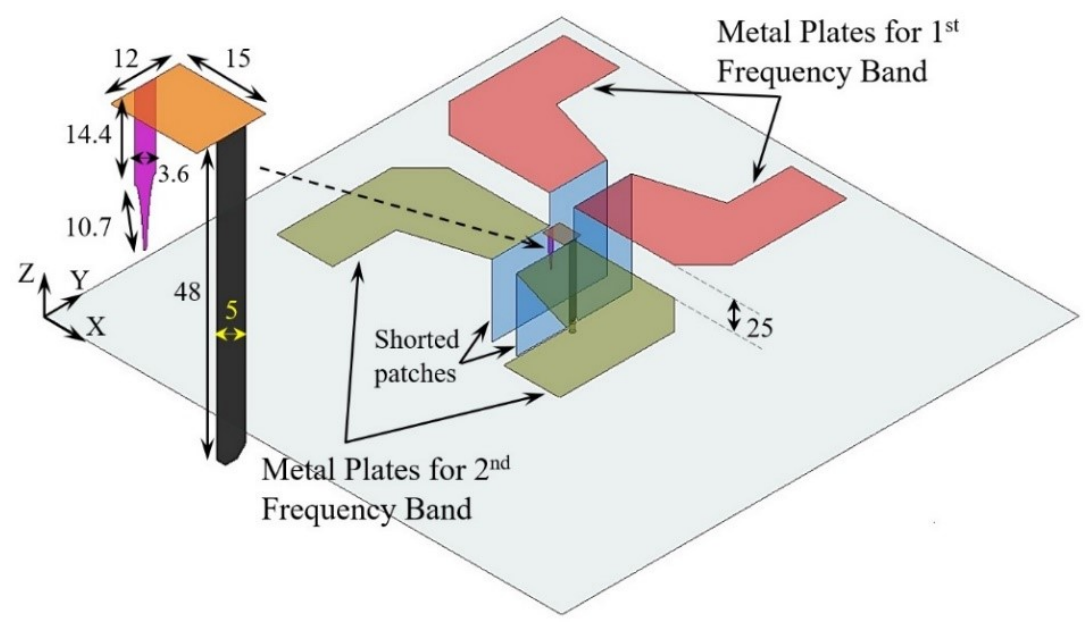

(a)

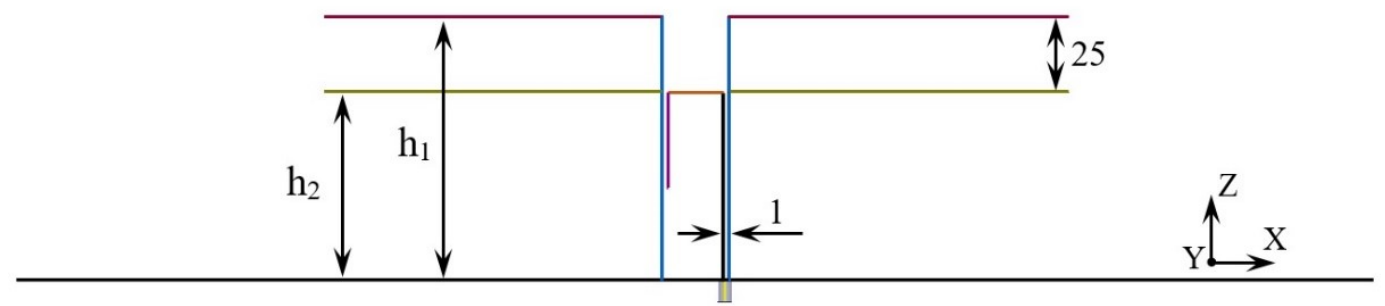

(b)

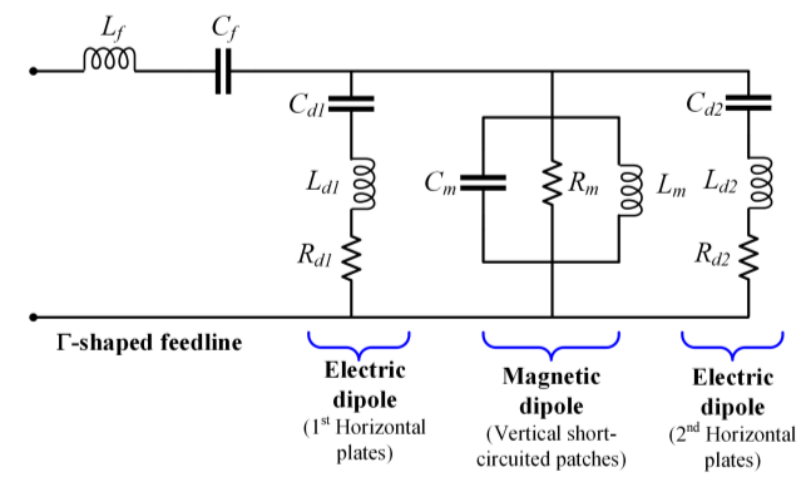

(c)

Fig. 4 Proposed dual-band MED antenna and $\Gamma$-shaped feed structure, a) Perspective view of the antenna and $\Gamma$ shaped feed strip, b) Side view of the antenna, \& c) Equivalent circuit of the dual-band MED antenna. (units: $\mathrm{mm}$ ) 


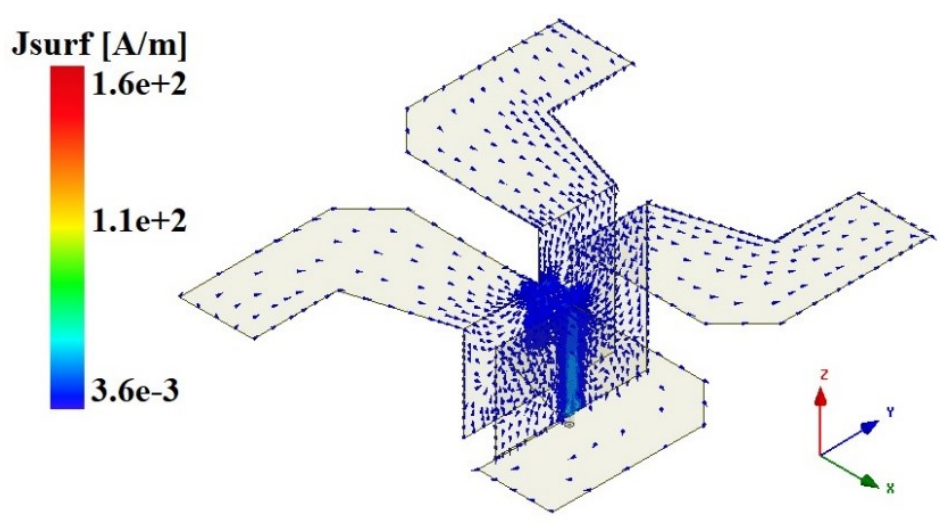

(a)

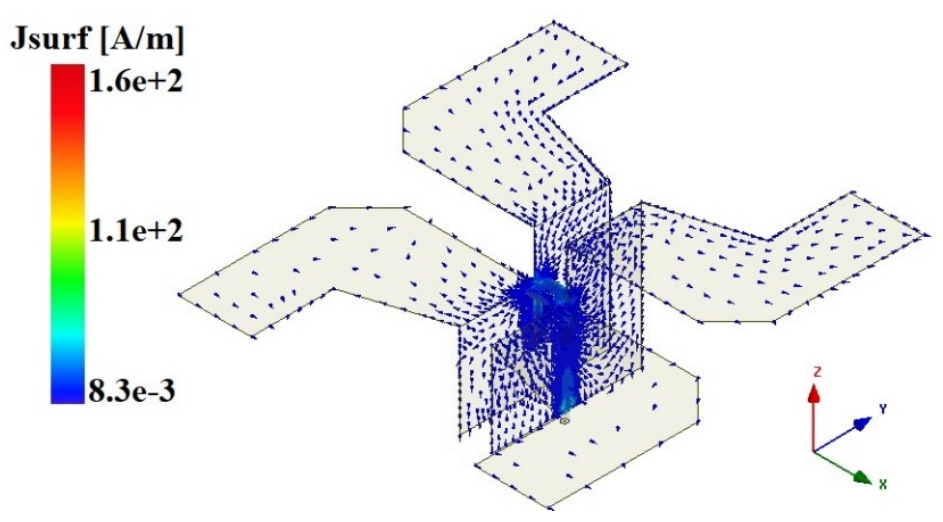

(b)

Fig. 5 Current distribution over the dual-band MED antenna, a) At $850 \mathrm{MHz}$, \& b) At $1800 \mathrm{MHz}$.

The optimized physical dimensions of the proposed dual-band antenna were obtained through parametric study using 3D electromagnetic simulation tool (HFSS. Ver13). The proposed dual-band MED antenna was fabricated in-house and tested using Agilent's Network Analyzer (E8363C). Fig. 6 shows photographs of the antenna in situ with the measurement setup. Simulated and measured $S_{11}$ response of the proposed antenna are shown in Fig.7(a). The numerical and measured results agree well with each other. According to the measurements, the proposed antenna achieves fractional bandwidth at the lower and upper bands of $13.31 \%$ (801.9 to $916.2 \mathrm{MHz}$ ) and $19.76 \%$ (1710.7 to $2085.7 \mathrm{MHz})$, respectively, for $\mathrm{S}_{11} \leq-10 \mathrm{~dB}$. The antenna gain is its ability to radiate efficiently relative to an isotropic antenna. Fig.7(b) shows the simulated and measured peak gain response of the antenna. The measured average peak gain is $9.4 \mathrm{dBi}$ at the first frequency band and $7.9 \mathrm{dBi}$ at the second band. The gain at the lower band is unusually higher than at the upper band. This is attributed to the better matching performance at the narrow lower band than at the upper band as evident in Fig.7(a). In the magneto-electric dipole antenna the gain is affected by the electromagnetic coupling between the two magneto-electric dipole segments. As the frequency of the signal increase in the operating band, the interaction of the shorter wavelength 
signals with the antenna segments increases and causes the gain to fall. This phenomenon is also observed in other published work on dual-band magneto-electric dipole antennas [7,8]. The antenna's radiation patterns in the H-plane ( $y z$-plane) and E-plane ( $x z$-plane) at 850 and $1800 \mathrm{MHz}$ are shown in Fig.8. The radiation patterns are stable and unidirectional with very low crosspolarization, and the antenna has a low backward radiation. The antenna's cross-polarization (Xpol) level is better than $-20 \mathrm{~dB}$ in both $\mathrm{H}$ - and E-planes at $850 \mathrm{MHz}$. At $1800 \mathrm{MHz}$, the $\mathrm{X}$-pol is better than $-20 \mathrm{~dB}$ in the H-plane and is approximately $-20 \mathrm{~dB}$ in the E-plane. The measured halfpower beamwidth (HPBW) is $105.4^{\circ}$ at $850 \mathrm{MHz}$ and $83.3^{\circ}$ at $1800 \mathrm{MHz}$.

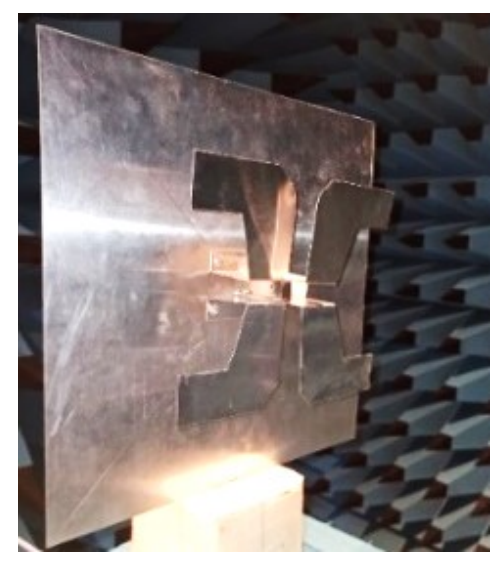

(a)

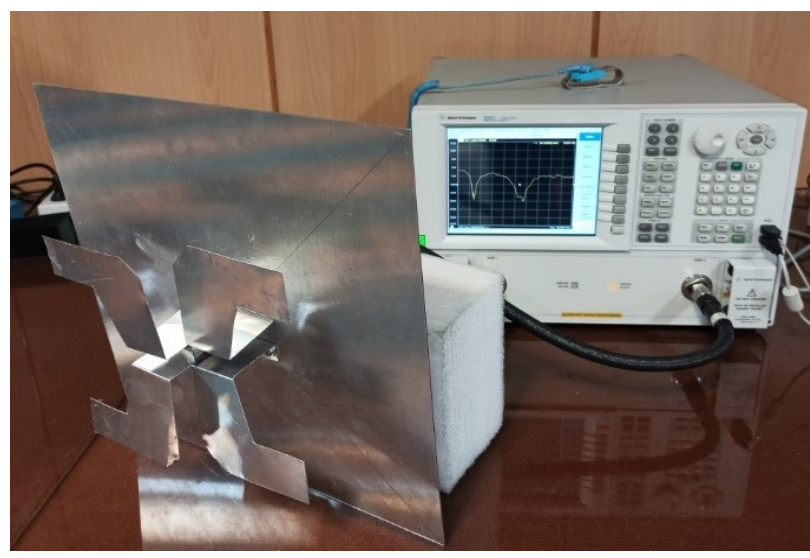

(b)

Fig. 6 Photographs of the fabricated dual-band MED antenna, a) The antenna in anechoic chamber, \& b) The antenna connected to the Network Analyzer.

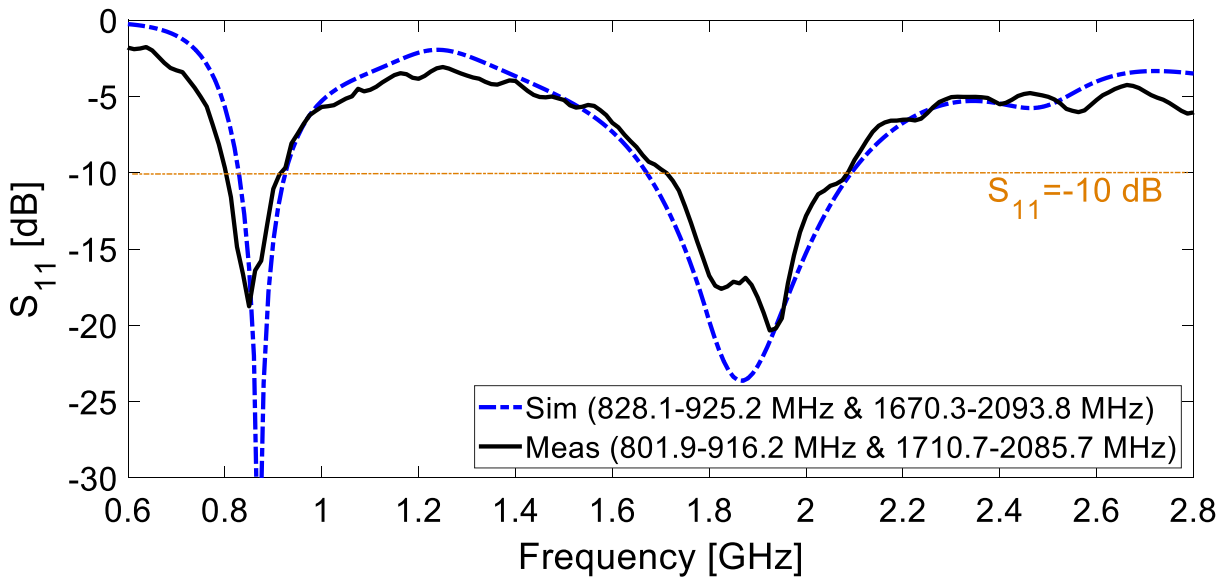

(a) 


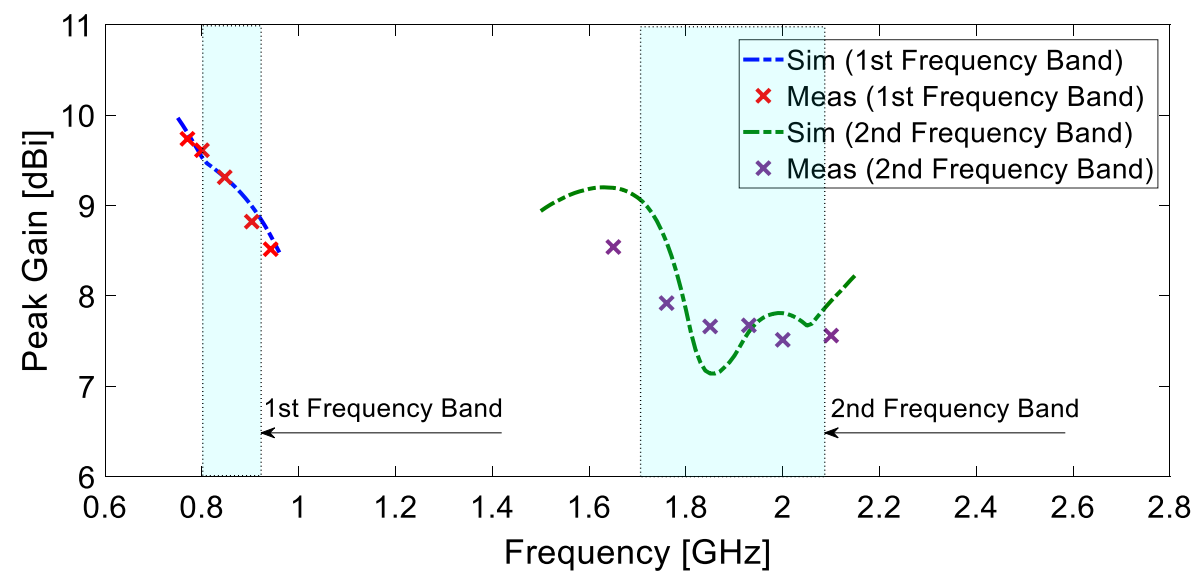

(b)

Fig. 7 Simulated and measured responses of the proposed dual-band MED antenna, a) $\mathrm{S}_{11}$ response, \& b) Peak gain at direction of $\phi=0^{\circ}$ and $\theta=0^{\circ}$.

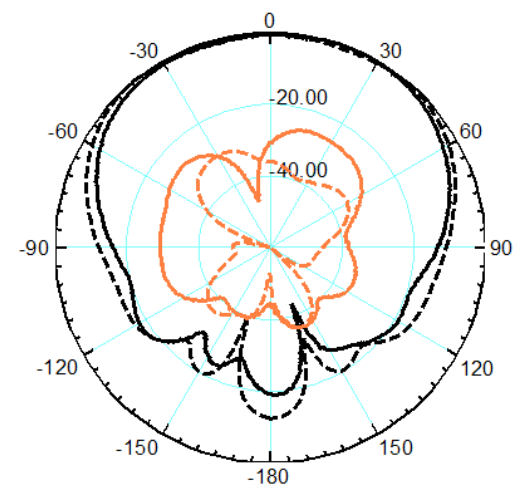

(a)

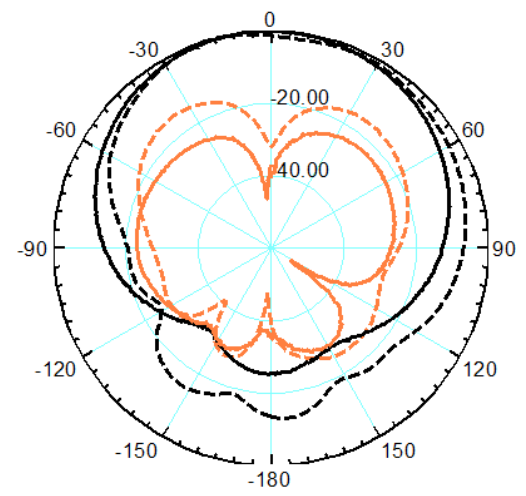

(c)

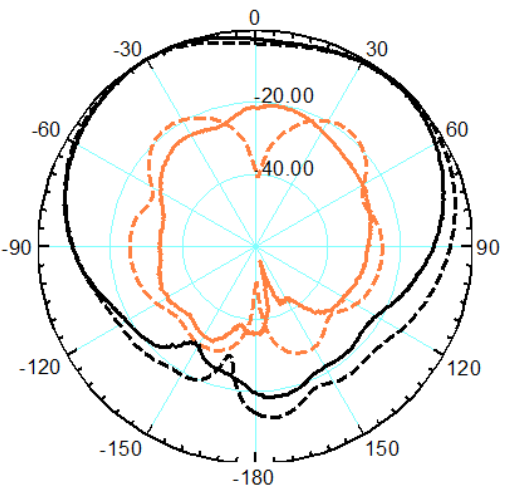

(b)

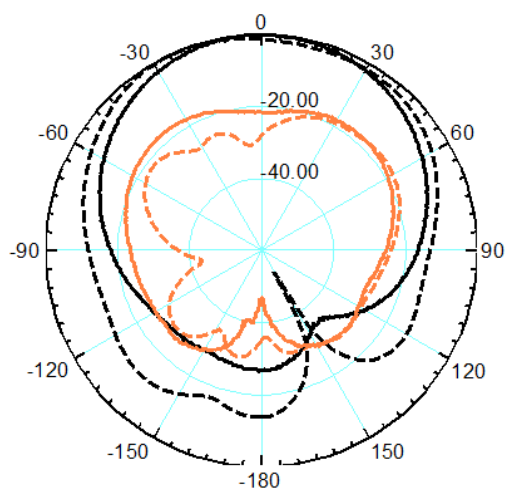

(d)

Fig. 8 Simulated and measured radiation patterns of the proposed dual-band MED antenna at 850 and $1800 \mathrm{MHz}$. a) $\mathrm{H}$-plane at $850 \mathrm{MHz}$, b) E-plane at $850 \mathrm{MHz}$, c) H-plane at $1800 \mathrm{MHz}$, \& d) E-plane at $1800 \mathrm{MHz}$. (Black lines represent $\mathrm{Co}$-pol and orange lines the X-pol. Measured radiation patterns are shown in solid lines, and simulated patterns in dash lines). 
Table 1 shows a comparison between the proposed antenna and other dual-band antennas in terms of operating impedance bandwidth, HPBW, broadside gain, and dimensions. Compared to other dual-band antennas the proposed design has the higher gain performance. In addition, the frequency ratio of the dual-band can be controlled by adjusting the gap between the two magnetoelectric dipole segments, and it is much simpler to design and manufacture.

Table 1. Size and performance comparison of dual-band MED antennas

\begin{tabular}{ccccc}
\hline Refs. & $\begin{array}{c}\text { Impedance BW } \\
(\mathrm{GHz}) / \text { FBW }(\%)\end{array}$ & $\begin{array}{c}\text { HPBW } \\
(\mathrm{deg})\end{array}$ & $\begin{array}{c}\text { Ave. Gain } \\
(\mathrm{dBi})\end{array}$ & $\begin{array}{c}\text { Dimensions } \\
\left(\lambda_{\mathrm{o}}{ }^{3}\right)\end{array}$ \\
\hline 6 & $2.13-2.62 / 20.6$ & 60 & 4 & $1.80 \times 1.80 \times 0.16$ \\
& $4.57-6.28 / 31.5$ & 104 & & \\
9 & $1.83-2.92 / 45.9$ & 30 & 7 & $0.95 \times 0.95 \times 0.24$ \\
& $2.39-2.52 / 5.29$ & 56 & & \\
10 & $0.80-1.10 / 31.6$ & $\mathrm{NR}$ & 3.6 & $0.64 \times 0.40 \times 0.0025$ \\
& $1.67-2.71 / 47.5$ & $\mathrm{NR}$ & & \\
11 & $1.68-2.93 / 54.2$ & 103 & 6.1 & $0.71 \times 0.70 \times 0.15$ \\
& $3.32-3.64 / 9.2$ & 165 & & \\
12 & $0.79-1.01 / 24.4$ & $\mathrm{NR}$ & 7.2 & $0.95 \times 1.36 \times 0.35$ \\
& $1.38-2.78 / 67.3$ & $\mathrm{NR}$ & 7.5 & \\
13 & $1.67-2.75 / 24.4$ & 49 & 5.9 & \\
& $3.22-4.13 / 24.7$ & 21.5 & 4.9 & \\
14 & $0.77-1.15 / 39.6$ & 90 & 4.4 & $0.97 \times 0.73 \times 0.88$ \\
& $1.66-2.93 / 55.3$ & 85 & 4.0 & \\
15 & $4.31-4.71 / 18$ & 84 & 4.4 & $0.73 \times 0.73 \times 0.11$ \\
& $5.07-5.89 / 45$ & 60 & 5.3 & \\
This & $0.80-0.92 / 13.4$ & 105.4 & 9.2 & $1.03 \times 1.03 \times 0.21$ \\
Work & $1.71-2.08 / 19.8$ & 83.3 & 7.8 & \\
\hline FBW & & & & \\
& & &
\end{tabular}

FBW: Fractional Bandwidth, NR: Not Reported

\section{Conclusion}

Design of an innovative high-gain dual-band MED antenna is verified by measurement. Dual-band functionality is created by employing two pairs of horizontal metal plates of different heights. The magneto-electric dipoles created by the two segments resonate at dual-band that is determined by the dimensions of the segments. The antenna exhibits impedance bandwidths of $13.31 \%$ centered at $850 \mathrm{MHz}$ and $19.76 \%$ centered at $1900 \mathrm{MHz}$ for $\mathrm{S}_{11} \leq-10 \mathrm{~dB}$. The gain performance in both operating bands is relatively high compared to other MED antennas. The proposed antenna 
exhibits stable radiation patterns and a low backward radiation, both in the lower and the upper band. The proposed dual-band MED antenna should find application in cellular base-stations.

\section{Acknowledgments}

The authors would like to acknowledge Dr. Barzegari for antenna fabrication and measurement in the Northwest Antenna and Microwave Research Laboratory (NAMRL) at Urmia University.

\section{References}

[1] Luk, K M, and Wong, H, “A new wideband unidirectional antenna element," Int. J. Microw. Opt. Technol., 2006, 1, (1), pp. 35-44.

[2] Liang W, Ni T, Li J, Jiao Y-C, "Circularly polarised magneto-electric dipole antenna," Electronics Letters 2014; 50:976-978. doi:10.1049/e1.2014.0812.

[3] Idayachandran G, Nakkeeran R, "Compact magneto-electric dipole antenna for LTE femtocell base stations,” Electronics Letters 2016; 52:574-576. doi:10.1049/el.2015.3266.

[4] Yang G, Zhang J, Zhou Z, "Low-profile broadband aperture coupled magneto-electric dipole array antenna for millimetre-wave applications," Electronics Letters 2020; 56:271-273. doi:10.1049/el.2019.3514.

[5] Yang M, Zhou J, Lian W, Chen B, "Dual-band dual-polarized magneto-electric dipole antenna with dual-layer structure," Progress in Electromagnetics Research M 2020; 92:193202. doi: 10.2528/pierm20022708.

[6] Yan S, Soh PJ, Vandenbosch GAE, "Wearable dual-band magneto-electric dipole antenna for WBAN/WLAN applications," IEEE Transactions on Antennas and Propagation 2015; 63:4165-4169. doi:10.1109/tap.2015.2443863.

[7] Yan S, Soh P.J., and Vandenbosch G.AA.E., "Wearable Dual-Band Magneto-Electric Dipole Antenna for WBAN/WLAN Applications," IEEE Trans. on Antennas \& Propagation, Vol. 63, No. 9, Sept. 2015:4165-4169.

[8] He K, Gong S-X, and Gao F., "A wideband dual-band magneto-electric dipole antenna with improved feeding structure," IEEE Antennas \& Wireless Propagation Letters, Vol. 13, 2014:1729-1732.

[9] Zhai H, Zhang J, Zang Y, Gao Q, Liang C, “An LTE base-station magnetoelectric dipole antenna with anti-interference characteristics and its MIMO System Application," IEEE Antennas and Wireless Propagation Letters 2015; 14:906-909. doi:10.1109/lawp.2014.2384519.

[10] Feng B, Yin S, Li S., "A planar-printed E-shaped omnidirectional magneto-electric dipole antenna for WWAN/LTE applications," Microwave and Optical Technology Letters 2014; 56:1734-1739. doi:10.1002/mop.28441. 
[11] Feng B, Chung KL, Lai J, Zeng Q., "A conformal magneto-electric dipole antenna with wide H-plane and band-notch radiation characteristics for sub-6-GHz 5G base-station," IEEE Access 2019; 7:17469-17479. doi:10.1109/access.2019.2896356.

[12] Feng B, Hong W, Li S, An W, Yin S., "A dual-wideband double-layer magnetoelectric dipole antenna with a modified horned reflector for $2 \mathrm{G} / 3 \mathrm{G} / \mathrm{LTE}$ applications," International Journal of Antennas and Propagation 2013; 2013:1-9. doi:10.1155/2013/509589.

[13] Lai J, Feng B, Zeng Q, Chung K. L., "A low-profile metamaterial magneto-electric dipole antenna with band-notch characteristics for 5-G MIMO base-stations," International Symposium on Antennas and Propagation (ISAP), 2017:1-2.

[14] Zhao Z, Lai J, Feng B, Sim C-Y-D, "A Dual-polarized dual-band antenna with high gain for 2G/3G/LTE indoor communications," IEEE Access, Vol. 6, 2018:61623-61632.

[15] Shuai C-Y, Wang G-M, "Substrate integrated low-profile dual-band magneto-electric dipole antenna," RF and Microwave Computer-Aided Engineering, Vol. 28, Issue 4, Dec. 2017:18 . 